\title{
VALORACIÓN DE LA CALIDAD DE ATENCIÓN A PACIENTES QUE SOLICITAN INTERRUPCIÓN TARDÍA DE LA GESTACIÓN
}

Assessing the Quality of Care for Patients Requesting a Late Termination of Pregnancy

Estela Moreno ${ }^{1-2}$, Sandra Suñol ${ }^{1}$, Yolanda Giménez ${ }^{1}$, Montse Palacio ${ }^{3}$, Ángela Arranz ${ }^{1-4}$, Olga Gómez ${ }^{3-5}$

Autora correspondencia: Estela Moreno

Correo electrónico: esmoreno@clinic.cat

1. Enfermera de BCNatal. Instituto de Ginecología, Obstetricia y Neonatología. Hospital Clínic de Barcelona. (Barcelona, España)

2. Enfermera de investigación. Instituto de Salud Global de Barcelona (ISGLOBAL). (Barcelona, España)

3. Especialista en Medicina Maternofetal. BCNatal - Barcelona Center for MaternalFetal and Neonatal Medicine, Hospital Clínic and Ho IDIBAPS, Universitat de Barcelona y Centre for Biomedical Research on Rare Diseases (CIBER-ER). (Barcelona, España)

4. Enfermera jefa del servicio de Enfermería de BCNatal (Hospital Clínic de BarcelonaHospital Sant Joan de Déu). (Barcelona, España)

5. Coordinadora del Grupo de duelo de BCNatal (Hospital Clínic de Barcelona-Hospital Sant Joan de Déu), Hospital Clínico de Barcelona. (Barcelona, España)

Recibido: 10/06/2021 Aceptado: 29/12/2021 


\section{RESUMEN}

OBJETIVO. Evaluar la experiencia de las pacientes en proceso de interrupción voluntaria del embarazo por malformaciones fetales graves o incurables por encima de las 22 semanas de gestación. MATERIAL Y MÉTODOS. Se realizó un estudio piloto descriptivo de corte transversal empleando un cuestionario ad hoc con 15 preguntas relacionadas con la calidad de atención y el proceso de pérdida gestacional desarrolladas por un equipo multidisciplinar del Hospital Clínic de Barcelona. La calidad de la atención se evaluó mediante una puntuación de 0 a 10 puntos. RESULTADOS. La puntuación global de la atención prestada fue de 9,45. El ruido ambiental en las diferentes instalaciones hospitalarias fue puntuado con un 5,98/10 y el tiempo desde el parto hasta el alta obtuvieron una puntuación de 6,22. A todas las pacientes se les ofreció contacto con su bebé, pero al $58 \%$ de las familias les hubiera gustado recibir además de las huellas otros recuerdos y al $47 \%$ hubiera deseado hacer fotografías a su bebé. Además, el $37 \%$ de las familias dijeron que hubiesen pasado más tiempo con su bebé. Finalmente, el $53 \%$ de las familias refirió la necesidad de requerir apoyo psicológico o suporte emocional, tanto durante el ingreso hospitalario como en el puerperio. CONCLUSIÓN. La valoración general de la atención prestada fue adecuada, aunque algunos aspectos podrían mejorarse. La realización de una encuesta a las familias sometidas a un IVE tardío es un método útil para evaluar la calidad de la atención brindada y diseñar acciones de mejora.

Palabras clave: atención de calidad; aborto; muerte fetal; duelo perinatal; atención hospitalaria.

\section{ABSTRACT}

OBJECTIVE To evaluate the experience of patients who were in the process of pregnancy interruption due to serious or incurable fetal malformations above 22 weeks' gestation. MATERIALS AND METHODS. A descriptive, cross-sectional pilot study was carried out using an ad-Hoc questionnaire that included 15 questions related to the quality of care and the gestational loss process developed by a multidisciplinary team of nurses and specialists in Maternal Fetal Medicine from the Hospital Clínic de Barcelona. The quality of the «care» was evaluated using a score from 0-10 points. RESULTS/FINDINGS. The global «Care» score was 9.45. The environmental noise score was 5.98 in the different hospital facilities and the perception of time from delivery to hospital discharge had a score of 6.22. All our patients were offered contact with their babies after the interruption, but $58 \%$ of the families would have liked to receive other regards in addition to the fingerprints they receive and $47 \%$ would have liked to have taken photographs of their babies. Additionally, $37 \%$ of families said they would have spent more time with them. Finally, $53 \%$ of the families reported the need to require psychological or emotional support, both during hospital admission and in the puerperium. CONCLUSIONS. The general assessment of the care provided was appropriate, although some aspects could be improved. Conducting a survey of families undergoing a late pregnancy interruption is a useful method to assess the quality of care provided and to design future actions.

Keywords: Quality Care; Abortion; Stillbirth; Perinatal Bereavement; Hospital Care. 


\section{INTRODUCCIÓN}

La atención hospitalaria a los padres que se enfrentan a una interrupción voluntaria del embarazo (IVE) por anomalías fetales graves o incurables es un proceso complejo en el que se viven momentos intensos en un corto período de tiempo que se inicia desde que se identifica la anomalía fetal y se realiza la evaluación pronóstica hasta el momento de la cuarentena tras el parto (Heazell et al., 2016; Nuzum et al., 2018). La IVE es un acto indeseable y representa una crisis que afecta a la mujer embarazada, pareja, familia y sociedad. En este período (Downe et al., 2013), los padres tienen que afrontar el diagnóstico, el duelo por la pérdida del hijo/a, la dificultad de tomar decisiones difíciles e importantes, las dudas sobre si conocer al bebé tras el parto, sobre cómo comunicar lo sucedido a sus familiares y amigos; y sobre todo cómo despedirse de él/ella (Flenady et al., 2014; Cassidy, 2018) y la incertidumbre de lo que pasará en el futuro.

El aborto es un tema controvertido, ya que toca aspectos religiosos, sociales y bioéticos, además de tópicos científicos y deontológicos que aún no han podido ser determinados, como lo es la definición misma de vida.

El duelo perinatal se define como el que se produce por la pérdida de un hijo/a antes o después del nacimiento, hecho que provoca un duelo que a menudo no es reconocido por la comunidad científica ni por la sociedad; se trata de un duelo «silenciado». El duelo perinatal afecta tanto si la pérdida se ha producido en las primeras semanas de gestación como después del nacimiento, independientemente de si la pérdida ha sido voluntaria o se ha producido de forma inesperada (Gómez et al., 2021).

La sociedad, familia, amistades y profesionales que atienden estas parejas pueden infravalorar y a menudo negar la existencia de este tipo de duelo. Esta actitud distante, de negación o de infravaloración hace que todavía sea más difícil para las parejas superar este proceso y genera una situación de malestar que dificulta avanzar en el proceso de duelo de forma saludable. Es por ello por lo que el Ministerio de Sanidad, Política Social e Igualdad en su Estrategia Nacional de Salud Sexual y Reproductiva (2011) recomienda el apoyo a las familias en situaciones de pérdida y duelo perinatal a través de los protocolos centrados en la atención a los aspectos humanos con el fin de respetar las necesidades individuales que expresan las mujeres y su/s acompañante/s. Entre las medidas que se proponen, se encuentra la posibilidad de ofrecer espacios físicos y tiempo de intimidad tras el parto, de ver y estar con el bebé y abrazarlo si los padres así lo desean, 
así como de respetar la decisión de no hacerlo; de conservar unos recuerdos del recién nacido y de referirse al bebé utilizando su nombre en caso de que los padres ya lo hayan decidido. Se remarca también la importancia de tratar de dar siempre una explicación sobre lo sucedido, respondiendo a las dudas todas las veces que sea necesario, involucrando a la madre y a su pareja en las decisiones respecto al destino del cuerpo del bebé, no minimizando el dolor de los padres, permitiendo que lo expresen sin juicio, mediante escucha empática y ofreciendo una ayuda psicológica en los casos en los que se necesite.

El cuidado de los padres que se enfrentan a una pérdida durante la gestación o tras el parto, incluyendo el apoyo emocional y psicológico, ha demostrado ser beneficioso para disminuir la ansiedad, la depresión y mejorar la vivencia global, convirtiendo un proceso negativo en una experiencia diferente con una nueva visión, como es la creación de recuerdos de un ser querido que, aunque aún no ha nacido, ya forma parte de la familia (Kingdon et al., 2015; Blood y Cacciatore, 2014). Aunque la implementación de estos cuidados debería ser ya obligatoria, aún no se ha extendido en todos los ámbitos.

Actualmente y debido a la evidencia descrita previamente, se han creado guías (Flenady et al., 2009; ACOG, 2009) y se han realizado diferentes encuestas para la valoración de la atención de la pérdida perinatal donde se ya se ha identificado la importancia del soporte emocional y psicológico además del físico, y sobre todo la interacción con el personal de salud (Aiyelaagbe et al., 2017; O’Connell et al., 2016).

El estudio de Cassidy (2018) es el único que podría describir la realidad en España. Se encontró que solo el $52 \%$ de las gestantes tuvieron contacto con sus bebés, el 30\% tuvo solo algún recuerdo y el 70\% estuvo acompañado. Sin embargo, esta encuesta, como las otras, es online. Posiblemente las encuestas presenciales puedan aportar más información y recoger de forma más completa los puntos posibles de mejora. Ese es el diseño planteado en nuestro estudio, en el que podemos explicar con detalle el contenido de la encuesta a las familias y damos la opción a las parejas de realizar el cuestionario en el momento en que ellos crean más conveniente.

Nuestro hospital constituye un centro de referencia de Patología Fetal, por lo que se atiende anualmente a un gran número de parejas que solicitan una IVE siguiendo el marco legal actual. Desde el año 2010, se han atendido más de 1300 parejas en el contexto de una IVE. El circuito asistencial que se sigue en nuestro centro se detalla en la Figura 1, 
mientras que la Figura 2 detalla el circuito de ingreso de la paciente una vez realizada la admisión en el hospital.

Aunque el protocolo de asistencia médica está plenamente validado y testado, en términos de una baja estancia media y una baja tasa de complicaciones, hasta el momento actual no existe un consenso general sobre el proceso de acompañamiento y soporte emocional en las diferentes áreas hospitalarias y entre los diferentes profesionales sanitarios, lo que constituye un punto de mejora futura. La pérdida de un hijo independientemente de la edad gestacional es una experiencia traumática para la familia. El cuidado continuo, el trato respetuoso y el apoyo emocional por parte de todos los profesionales durante todo el proceso son fundamentales para la elaboración de un proceso de duelo no patológico.

Figura 1. Circuito de interrupción voluntaria de embarazo en nuestro centro

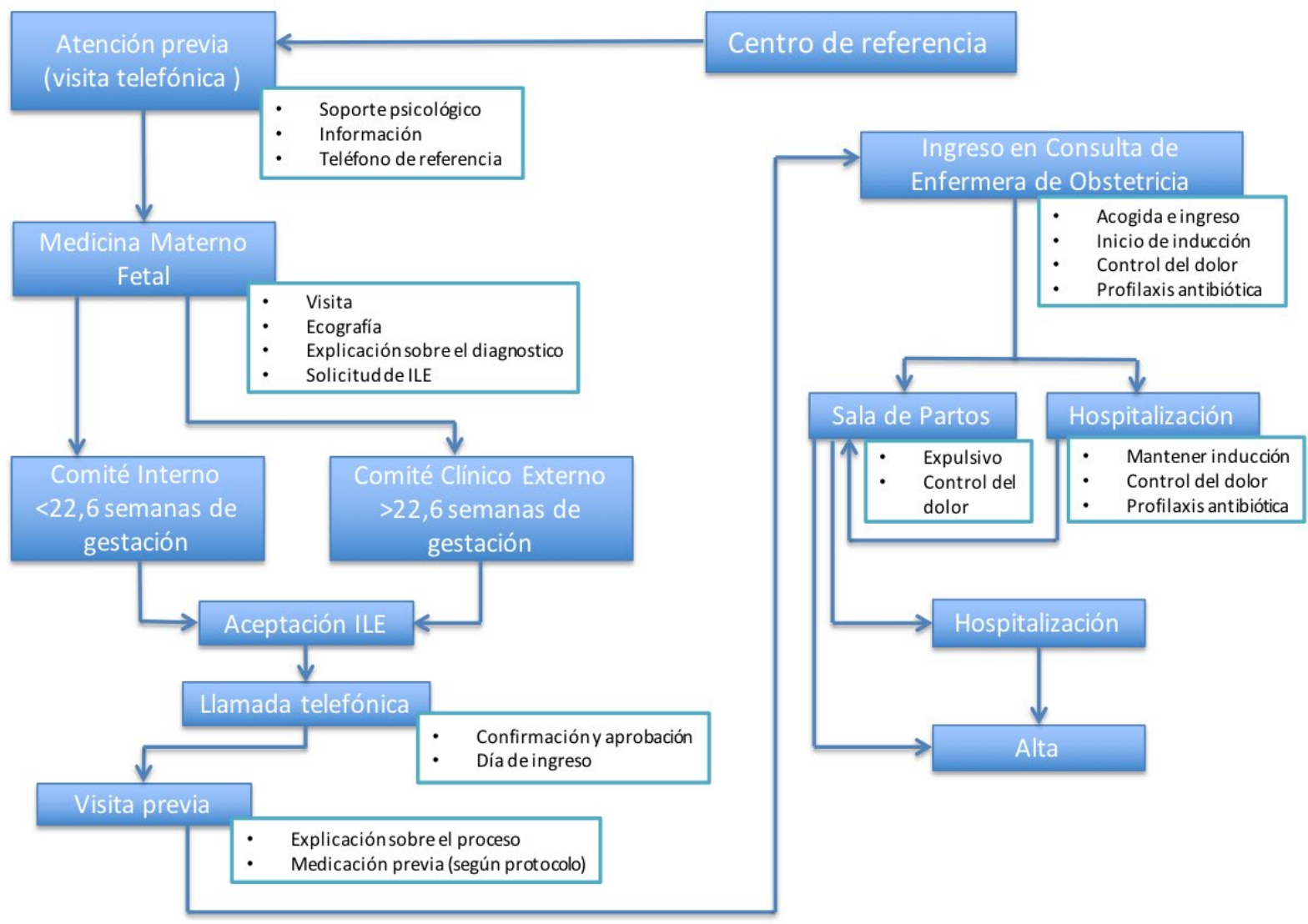

Fuente: Elaboración propia. 
Figura 2. Circuito de ingreso de la paciente

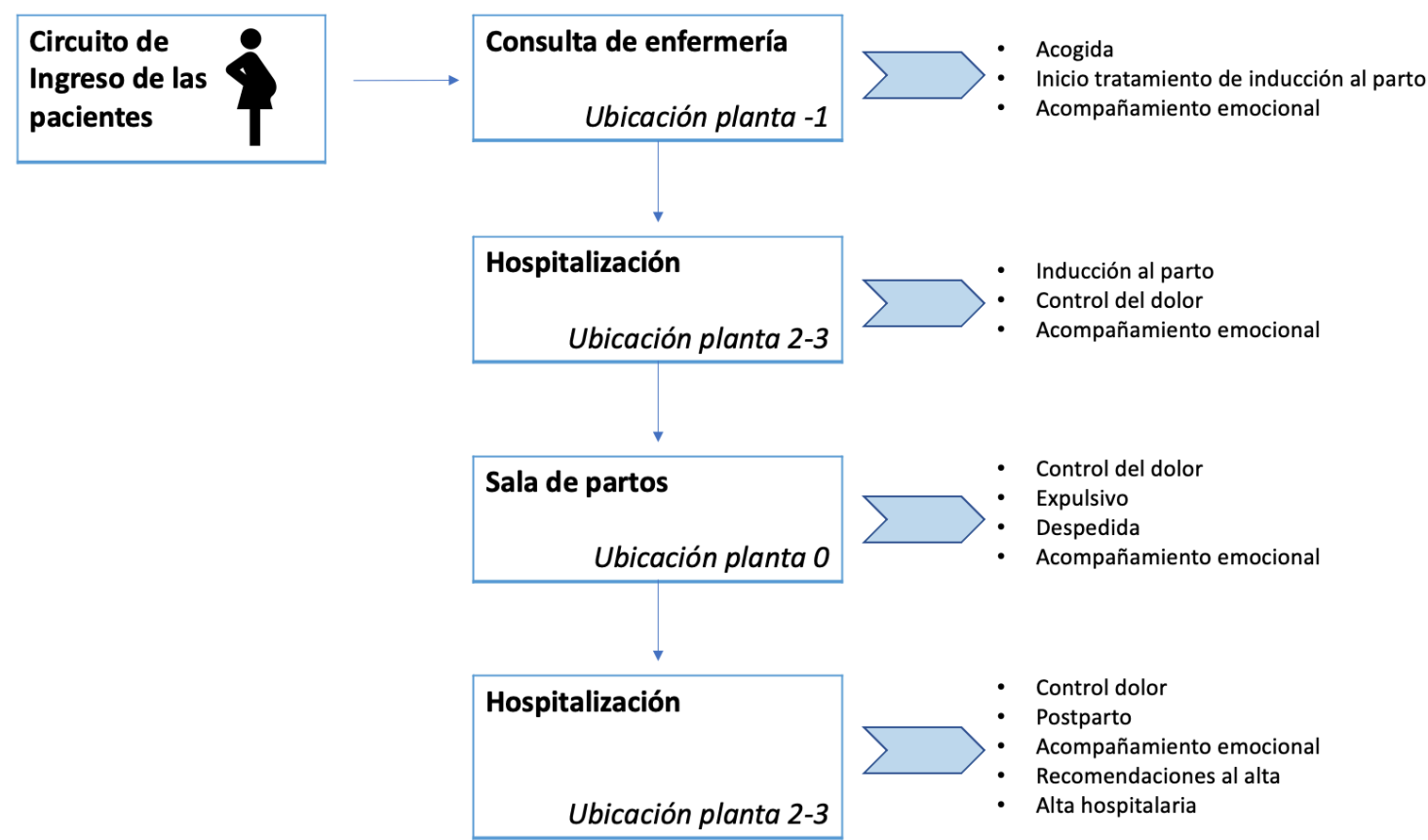

Fuente: Elaboración propia.

Este proyecto surgió tras conocer las necesidades que nos verbalizaron nuestras pacientes durante la práctica clínica diaria habitual. El procedimiento de una IVE tardía en el contexto de una patología fetal es un proceso largo y complejo. Desde que se diagnostica la patología fetal hasta que finaliza todo el proceso después de la cuarentena, las familias pasan por múltiples áreas del hospital (consultas de ecografías, consulta de enfermería obstétrica, sala de partos, hospitalización), y también por múltiples profesionales sanitarios (enfermeras, matronas, auxiliares de enfermería, especialistas en medicina materno-fetal, anestesiólogos, celadores, administrativos, personal de limpieza...). Así, la coordinación de todo el proceso genera disfunciones que repercuten en la percepción de la calidad del trato recibido y que son a su vez mejorables si se detectan adecuadamente. El presente trabajo constituye el primero realizado en nuestro centro en que se evalúa la IVE tardía, colocando la vivencia de la paciente en el centro de todo el proceso.

Por ello, la finalidad de este trabajo es evaluar la experiencia de las pacientes en proceso de IVE por malformaciones fetales graves o incurables por encima de las 22 semanas de gestación evaluando las medidas de acompañamiento y soporte emocional que se realizan en nuestro centro mediante la implementación de unas encuestas diseñadas con el propósito de poder analizar los resultados obtenidos para mejorar las prácticas del 
personal de salud, homogeneizar la actuación de los diferentes profesionales, así como fortalecer la confianza entre el equipo multidisciplinario y la mujer para ofrecer un trato digno, adecuando las medidas actuales para conseguir un proceso más eficiente centrado en las familias y adaptándolo a sus necesidades reales.

\section{MATERIAL Y MÉTODOS}

Se realizó un estudio piloto descriptivo de corte transversal empleando un cuestionario ad hoc conformado por 15 preguntas relacionadas con la calidad de la atención y el proceso de pérdida gestacional/interrupción de la gestación en el Hospital Clínic de Barcelona-Sede Maternidad entre marzo y agosto de 2019.

El equipo investigador valoró y decidió la realización de este estudio con carácter exploratorio para valorar el proceso de atención de la pérdida/interrupción de la gestación con el objetivo general de plantear mejoras en la calidad de atención y/o servicios relacionados con un proceso tan delicado como este y el cual está poco estudiado. A fin de llevar a cabo este proceso, se elaboró una encuesta ad hoc para objetivar la calidad de atención prestada en diferentes ámbitos como infraestructuras, trato, valoración de circuito y apoyo emocional, entre otros.

Para ello se realizó la encuesta a mujeres en proceso de interrupción de la gestación que cumplieron los criterios de inclusión y exclusión, y que accedieron voluntariamente y de forma anónima a participar en el estudio en el momento del alta hospitalaria.

Con el fin de poder incluir a las pacientes en el estudio y poder recoger los datos necesarios para su evaluación, se informó inicialmente de forma verbal sobre el estudio, sus objetivos y posibles beneficios. Posteriormente se obtuvo el consentimiento informado, respetando la privacidad y la confidencialidad de las respuestas, siendo únicamente utilizadas para fines de la investigación, cumpliendo siempre con la confidencialidad y la ética de los profesionales, siguiendo los principios bioéticos establecidos en la Declaración de Helsinki. Las pacientes tenían la posibilidad de abandonar el estudio en cualquier momento si así lo expresaban.

Los criterios de selección del estudio fueron los siguientes:

- Criterios de inclusión:

- Gestantes que soliciten un ILE $\geq 22$ semanas de gestación 
- Gestantes mayores de 18 años

- Criterios de exclusión:

- Gestantes menores de edad

- Barrera idiomática (disponemos de cuestionarios en catalán, castellano e inglés)

- Gestaciones múltiples con interrupción selectiva de uno de los fetos

El reclutamiento se realizó de forma presencial durante el ingreso de las gestantes en proceso de IVE por parte del equipo investigador. Se seleccionó una muestra de 20 pacientes que corresponde al $50 \%$ de las gestantes atendidas en nuestro centro durante un año. Dicha muestra se calculó con el programa STATSTM versión 2.0, con un índice de confianza de $95 \%$ y un margen de error menor al $5 \%$.

La encuesta utilizada fue desarrollada por un equipo multidisciplinar del Hospital Clínic de Barcelona conformado por ginecólogos especialistas en materno-fetal, enfermeras especialistas en obstetricia, neonatólogos y comadronas. En la encuesta se valoró la «calidad de la atención» y el proceso de interrupción de la gestación que se lleva a cabo en nuestro hospital.

La encuesta se estructuró de la siguiente manera (Tabla 1):

1. Preguntas para valorar los espacios físicos/infraestructuras hospitalarias, los profesionales sanitarios y la calidad en la atención recibida (preguntas 1-6).

2. Preguntas para valorar las necesidades de los pacientes con respecto a la intimidad durante el proceso de parto hasta el alta, sobre su opinión respecto a la idea de disponer de recuerdos del recién nacido sin vida y también sobre la valoración de las prácticas recibidas durante todo el proceso (preguntas 7-11 y pregunta 15).

3. Preguntas para valorar la información recibida durante el proceso, el apoyo emocional y la idoneidad de realizar el cuestionario recibido (preguntas 12-14).

El diseño de la encuesta se realizó mediante un sistema de valoración cuantitativa (utilizando una escala numérica del 1 al 10). Una puntuación de «cero» representa una mala valoración mientras que la puntuación de «diez» una valoración excelente. Todas las preguntas relacionadas con las infraestructuras hospitalarias tenían la opción de respuesta abierta, con el objetivo de que las encuestadas tuviesen un espacio para resaltar 
algún aspecto importante no mencionado con anterioridad. Una vez finalizada la encuesta, había una pregunta abierta para que las familias que lo crean necesario puedan expresar sugerencias, recomendaciones o bien agradecimientos al personal.

Toda la información recogida se almacenó de forma confidencial en nuestro centro cumpliendo la normativa vigente y, a posteriori, se introdujeron los datos cuantitativos en un programa estadístico (base datos en Microsoft Excel) para su análisis.

El análisis descriptivo de las respuestas de la encuesta, así como las diferentes mediciones se realizó utilizando el programa SPSS IBM 2016. Los datos cuantitativos fueron presentados con promedios y rangos, mostrando los valores máximos y mínimos para cada una de las preguntas cuantitativas. En el caso de las preguntas abiertas, se realizó un análisis textual.

El acceso y análisis de la base de datos quedó restringida a los investigadores principales: la Srta. Estela Moreno y Dra. Olga Gómez.

Tabla 1. Distribución de las preguntas de la Encuesta ad hoc

\begin{tabular}{|l|c|l|}
\hline Indicadores & $\begin{array}{l}\text { Preguntas del } \\
\text { cuestionario }\end{array}$ & Valores posibles \\
\hline $\begin{array}{l}\text { Infraestructuras } \\
\text { hospitalarias }\end{array}$ & $1-2-3-4$ & Variable cuantitativa (puntuación 1-10) \\
\hline Calidad de atención & $5-6$ & Variable cuantitativa (puntuación 1-10) \\
\hline Creación de recuerdos & $7-8-9-10-11-$ & $\begin{array}{l}\text { Variables dicotómicas } \\
\text { (Sí/No) }\end{array}$ \\
\hline Circuito administrativo & 15 & Variable cualitativa nominal \\
\hline Información & 13 & $\begin{array}{l}\text { Variables dicotómicas } \\
\text { (Sí/No) }\end{array}$ \\
\hline Apoyo psicológico & 14 & $\begin{array}{l}\text { Variables dicotómicas } \\
\text { (Sí/No) y variable cualitativa nominal }\end{array}$ \\
\hline Pregunta abierta & 15 & Análisis textual \\
\hline
\end{tabular}


El proyecto fue presentado, evaluado y aceptado por el Comité de Ética de Investigación (CEIC) del Hospital Clínic con código de aceptación HCB/2019/012. Por otro lado, los resultados obtenidos en el mencionado estudio no implicaron ningún cambio en la actitud terapéutica ni en el seguimiento médico estándar de la paciente.

\section{RESULTADOS}

La Tabla 2 hace referencia al resumen de las puntuaciones obtenidas en relación con la calidad de la atención recibida. Los resultados obtenidos sobre atención recibida por los distintos profesionales de las diferentes unidades han sido muy favorables, obteniendo una $\bar{x}$ de 9.45, que ha sido obtenido de la suma total de las diferentes unidades analizadas en diferentes áreas incluyendo consultas de medicina materno-fetal, consulta de enfermería, sala de partos y hospitalización, debido a que las pacientes deben moverse por distintas áreas del hospital como se muestra en la Figura 2.

También se analizó la percepción sobre del proceso recibido con una $\overline{\mathrm{x}} 8.65$ en las áreas de explicación sobre el proceso, atención, acompañamiento, apoyo y gestión emocional.

Tabla 2. Cuestionario de valoración de la calidad de atención percibida por los profesionales en la IVE

\begin{tabular}{|l|l|c|}
\hline Pregunta & Características Valoradas & $\begin{array}{l}\text { Puntuación } \\
\text { media +/- DE }\end{array}$ \\
\hline \multirow{2}{*}{$\begin{array}{l}\text { Valoración de la atención recibida } \\
\text { por los profesionales }\end{array}$} & $\begin{array}{l}\text { Consulta externa (CCEE) Unidad } \\
\text { materno-fetal }\end{array}$ & $9,65+/-0,58$ \\
\cline { 2 - 3 } & CCEE Enfermería & $9,79+/-0,41$ \\
\cline { 2 - 3 } & Sala de partos & $9,61+/-0,97$ \\
\cline { 2 - 3 } por parte de todo el equipo que le & Sala de Hospitalización & $8,75+/-1,65$ \\
\cline { 2 - 3 } ha atendido & Atención durante el proceso & $8,25+/-2,33$ \\
\cline { 2 - 3 } & Acompañamiento durante el & $8,85+/-1,38$ \\
& proceso & $8,6+/-1,75$ \\
\cline { 2 - 3 } & Apoyo y gestión emocional & \\
\hline
\end{tabular}


En la Tabla 3, se muestran las valoraciones obtenidas sobre los espacios físicos. Se describen las respuestas sobre las necesidades de los pacientes con respecto a la intimidad durante el proceso de parto hasta el alta, espacios, confort, ruidos ambientales, ubicación y tiempos de espera. Estos análisis fueron examinados de nuevo en las diferentes áreas mencionadas: consultas de medicina materno-fetal, consulta de enfermería, sala de partos y hospitalización.

Tabla 3. Cuestionario de valoración de los espacios físicos del proceso de pérdida perinatal

\begin{tabular}{|c|c|c|}
\hline Pregunta & Características Valoradas & $\begin{array}{l}\text { Puntuación } \\
\text { media +/-DE }\end{array}$ \\
\hline \multirow{4}{*}{$\begin{array}{l}\text { Valoración de la consulta donde se le } \\
\text { explicó el pronóstico de su bebé }\end{array}$} & Espacio & $8,79+/-1,43$ \\
\hline & Intimidad & $8,74+/-1,48$ \\
\hline & Confort & $8,21+/-1,51$ \\
\hline & Ruidos Ambientales & $7,68+/-2,53$ \\
\hline \multirow{5}{*}{$\begin{array}{l}\text { Valoración de la consulta de } \\
\text { enfermería }\end{array}$} & Espacio & $8,3+/-1,56$ \\
\hline & Intimidad & $8+/-2,45$ \\
\hline & Ubicación & $6,05+/-3,25$ \\
\hline & Ruidos Ambientales & $5,05+/-3,79$ \\
\hline & Tiempo de espera a ser atendido & $9,73+/-1$ \\
\hline \multirow[t]{6}{*}{ Valoración de sala de partos } & Espacio & $9,33+/-0,84$ \\
\hline & Intimidad & $8,44+/-1,88$ \\
\hline & Ubicación & $8,06+/-2,86$ \\
\hline & Ruidos Ambientales & $5,94+/-4,07$ \\
\hline & Confort & $8,33+/-2$ \\
\hline & $\begin{array}{l}\text { Tiempo de espera al ingreso en sala } \\
\text { de partos }\end{array}$ & $8,94+/-1,39$ \\
\hline \multirow[t]{5}{*}{ Valoración de sala de hospitalización } & Espacio & $9,32+/-1,29$ \\
\hline & Intimidad & $8,65+/-2,16$ \\
\hline & Confort & $8,05+/-2,83$ \\
\hline & Ruidos Ambientales & $5,26+/-4,09$ \\
\hline & Tiempo de espera al alta & $6,22+/-3,13$ \\
\hline
\end{tabular}

Las valoraciones de la consulta de medicina fetal fueron positivas, con una $\overline{\mathrm{x}} 8,35$. Sin embargo, los ruidos ambientales fueron los peor valorados con 7,68+/-2,53. En la consulta de enfermería, la $\bar{x}$ fue de 7,43 , siendo peor valorada la ubicación con $6,05+/-$ 
3,25 y los ruidos ambientales con 5,05 +/-3,79. La sala de partos recibió buenas valoraciones con una $\bar{x} 8,17$, siendo en la misma, peor valorados también los ruidos ambientales con 5,94+/-4,07. Por último, la valoración de hospitalización fue de 7,5, resaltando de nuevo los ruidos ambientales con 5,26+/-4,09, y el tiempo de espera al alta una vez ha transcurrido el parto que es de 6,22+/-3,13 horas.

En la Tabla 4, se muestran las valoraciones en base a la creación de recuerdos. Se describen las respuestas sobre la idea de disponer de recuerdos del recién nacido sin vida y también sobre la valoración de las prácticas recibidas durante todo el proceso.

Tabla 4. Cuestionario de valoración relacionado con la creación de recuerdos

\begin{tabular}{|c|c|c|}
\hline Pregunta & Respuesta & Resultado n (\%) \\
\hline \multirow{2}{*}{$\begin{array}{l}\text { ¿Se le ofreció tener contacto con su bebé en sala de } \\
\text { partos? }\end{array}$} & Sí & $19(100)$ \\
\hline & No & $0(0)$ \\
\hline \multirow[t]{2}{*}{ ¿Se le ofreció obtener las huellas de su bebé? } & Sí & $19(100)$ \\
\hline & No & $0(0)$ \\
\hline \multirow{2}{*}{$\begin{array}{l}\text { ¿Le hubiera gustado algún otro recuerdo de su bebé } \\
\text { (gorro, pulsera, pinza del cordón)? }\end{array}$} & Sí & $11(58)$ \\
\hline & No & $8(42)$ \\
\hline \multirow[t]{2}{*}{ ¿Le hubiera gustado haber podido hacerle fotos? } & Sí & $9(47)$ \\
\hline & No & $10(53)$ \\
\hline \multirow{2}{*}{$\begin{array}{l}\text { ¿Considera que el tiempo que ha pasado con su bebé en } \\
\text { Sala de Partos ha sido suficiente? }\end{array}$} & Sí & $10(63)$ \\
\hline & No & $6(37)$ \\
\hline \multirow{2}{*}{$\begin{array}{l}\text { ¿Les hubiera gustado recibir el certificado de } \\
\text { nacimiento y el recuerdo de las huellas que proponemos } \\
\text { en nuestro centro? }\end{array}$} & Sí & $14(74)$ \\
\hline & No & $5(26)$ \\
\hline
\end{tabular}

A todos los pacientes se les ofreció el contacto con el recién nacido, así como la obtención de las huellas en una cartulina a modo de recuerdo. El momento en que se ofreció la posibilidad de obtener las huellas del recién nacido fue en consultas externas (43\%), en sala de partos (31\%) y, en el resto de los casos, el ofrecimiento se realizó en ambos sitios. El 58\% de los pacientes hubiera preferido, además de las huellas del recién nacido, disponer de otro recuerdo de su bebé, y el $47 \%$ hubiera deseado haberle hecho fotos. E1 $63 \%$ de las familias considera que el tiempo que pasó con su bebé fue adecuado. La propuesta de recibir un certificado de nacimiento elaborada por nuestro grupo de 
trabajo, así como el recuerdo personalizado de huellas, fue bien recibido por el $74 \%$ de pacientes.

La Tabla 5 muestra las respuestas en base a la información sobre los trámites administrativos recibidos. Se evidencia que a un $35 \%$ de las pacientes les hubiera gustado recibir la información respecto a los trámites administrativos con mayor detalle antes de la inducción del parto, a un 10\% durante el ingreso después del expulsivo, a un 50\% antes del alta y a 5\% les parecía indiferente el momento de recibir dicha información. También se analiza si las pacientes necesitan más información sobre el duelo perinatal y la idoneidad de realizar el cuestionario. Un 55\% de las madres considera que la información recibida al alta sobre los primeros pasos del duelo fue insuficiente.

Nuestro cuestionario fue cumplimentado durante la hospitalización de las pacientes. Sin embargo, la mayoría de las pacientes (70\%) hubiera preferido que el cuestionario se hubiera realizado durante la cuarentena, y de forma presencial el 30\%.

Tabla 5. Cuestionario de valoración sobre los trámites administrativos e información adicional

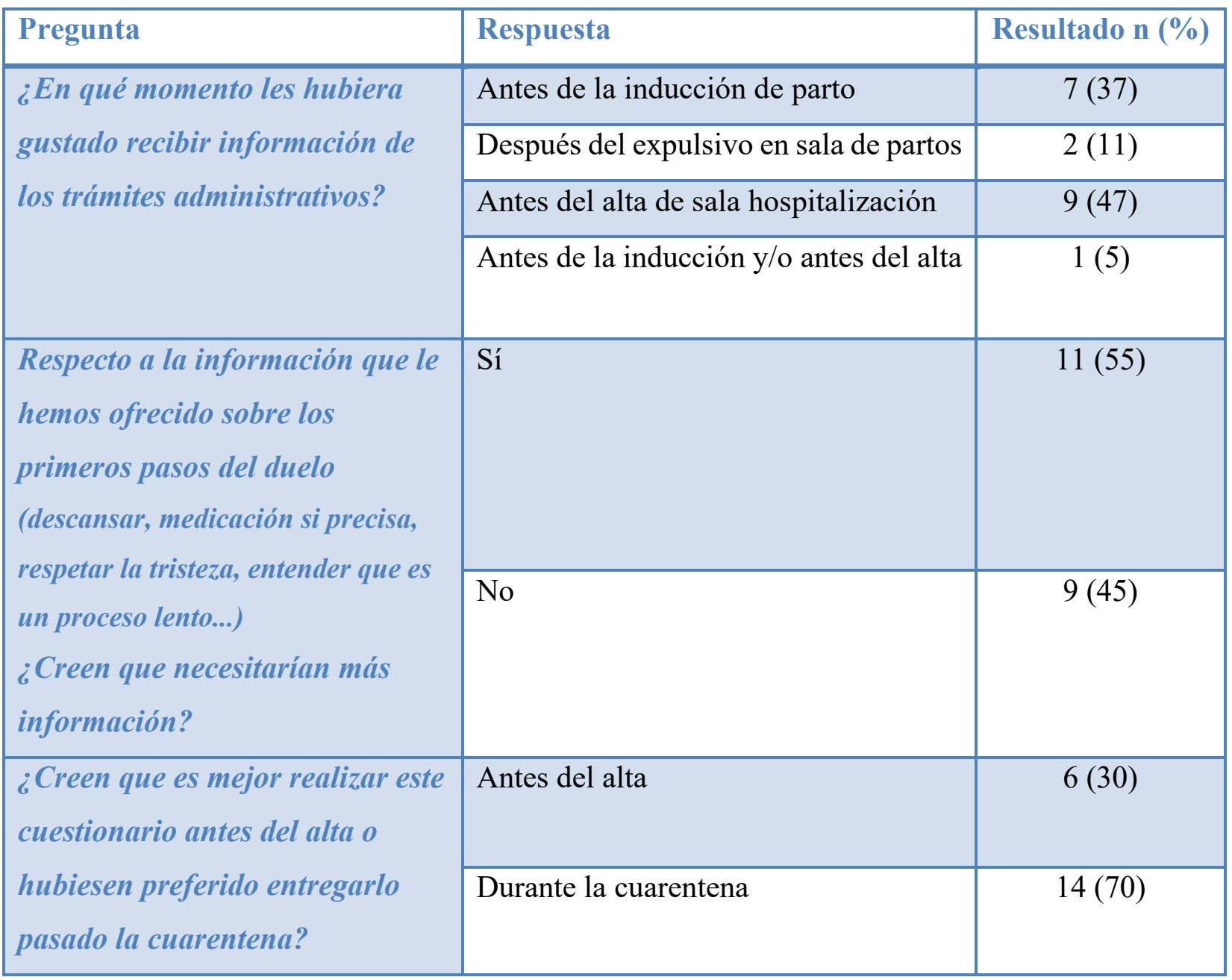


Y, por último, la Tabla 6 resume las respuestas obtenidas respecto al apoyo psicológico. El 53\% respondió que sería útil recibir más soporte emocional/psicológico. De estos, el 60\% manifestó su preferencia en forma de atención psicológica individualizada, el 20\% en forma de grupos de ayuda y el otro $20 \%$ contestó que preferiría ambos tipos de ayuda a la vez. Por otro lado, los momentos más adecuados para recibir la ayuda psicológica según las encuestadas fue durante el ingreso y tras la cuarentena $50 \%$.

Tabla 6. Cuestionario de valoración sobre la necesidad de apoyo psicológico

\begin{tabular}{|c|c|c|}
\hline Pregunta & Respuesta & Resultado n (\%) \\
\hline \multirow[b]{2}{*}{$\begin{array}{l}\text { ¿CREE QUE SERÍA ÚTIL O } \\
\text { NECESARIO RECIBIR MÁS } \\
\text { SOPORTE EMOCIONAL / } \\
\text { PSICOLÓGICO DURANTE EL } \\
\text { PROCESO? }\end{array}$} & Sí & $10(53)$ \\
\hline & No & $9(47)$ \\
\hline \multirow[t]{3}{*}{ a) ¿Qué tipo de ayuda desearía? } & Grupo de ayuda & $2(20)$ \\
\hline & Atención psicológica & $6(60)$ \\
\hline & Ambos & $2(20)$ \\
\hline \multirow{5}{*}{$\begin{array}{l}\text { b) ¿Qué momento sería el más } \\
\text { apropiado? }\end{array}$} & En el diagnóstico & $1(10)$ \\
\hline & $\mathrm{Al}$ ingreso/alta & $1(10)$ \\
\hline & Tras la cuarentena & $2(20)$ \\
\hline & $\begin{array}{l}\text { Al ingreso y tras la } \\
\text { cuarentena }\end{array}$ & $5(50)$ \\
\hline & Todos & $1(10)$ \\
\hline
\end{tabular}

En las preguntas abiertas, las familias expresaron aspectos importantes no recogidos en las preguntas realizadas por el equipo investigador. Algunas familias redactaron sugerencias y agradecimientos al equipo que las atendió. Los diferentes agradecimientos fueron trasladados a todo el equipo asistencial.

Se recogieron diferentes anotaciones relacionadas con el equipamiento y espacio de consultas externas donde se inicia todo el proceso, recomendando modernización y ampliación de espacio. Una paciente expresó que «Estuve mucho tiempo en una camilla 
incómoda», mientras que otra que «la sala de inicio del proceso es muy pequeña y poco confortable».

Por otro lado, varias encuestas $(n=5)$ sugirieron que se debería tener un acceso directo a sala de partos, evitando así el ingreso por consultas externas y posteriormente hospitalización. Una paciente escribió: «Recuerdo con muy mal sabor de boca los traslados por el propio hospital. Creo que para próximas familias que pasen por la misma situación que nosotros, deberían de ingresar directamente a sala de partos, evitando así los traslados por las distintas unidades del servicio», mientras que otra paciente refirió que «Me dolía ver continuamente a embarazadas felices con su gestación. Creo que se debería de evitar los traslados».

Otro aspecto de gran importancia detectado en las preguntas abiertas fue la insonorización acústica de sala de partos y hospitalización. El 35\% de las familias describen esta necesidad. Es así como una madre refirió que «Escuchar otros bebés llorar fue lo más duro para nosotros», o «tener a mi bebé sin vida en las manos y escuchar otros bebés nos partió el corazón», mientras que otra paciente escribió: «Consideramos que se debería de tener un espacio insonorizado para todas las familias que pasamos por un proceso de pérdida».

Por último, en dos ocasiones las pacientes han manifestado que se han sentido acosadas por el hecho de decidir no tener contacto con su bebé. Comentaron que en todos los servicios le han preguntado si quieren ver/conocer a su bebé y de cierta manera se han sentido mal por no estar preparados para ello. Así, una de ellas escribió: «Nos preguntaron muchas veces si queríamos conocer al bebé», mientras que la otra paciente refirió que «Teníamos muy claro que no lo queríamos ver y nos sentíamos con la obligación, ya que nos preguntaron cuatro veces lo mismo».

\section{DISCUSIÓN}

El manejo del duelo perinatal ha ido cambiando de forma muy significativa en los últimos años. A modo de ejemplo, en los años setenta se recomendaba que la madre evitará tener contacto con el bebé fallecido después del parto con la idea de que ello favorecía su recuperación posterior evitando su sufrimiento (O’Connell et al., 2016). Sin embargo, en los últimos años estas prácticas han ido cambiando (Downe et al., 2013) a medida que se ha ido evidenciando que el contacto con el bebé, la creación de recuerdos y la planificación de una despedida, entre otros, ayudan a mejorar el proceso de duelo en los 
padres, dejando atrás el estigma y el sentimiento de culpa de la madre, por un lado, y la ausencia del reconocimiento del propio bebé como persona, por otro (Frøen et al., 2011). El entorno particular de cada paciente y familia (Blood y Cacciatore, 2014) es también importante para la buena atención, evitando ejercer una presión excesiva en una dirección u otra (Erlandsson et al., 2013). Es por ello necesario entender las necesidades de las familias que han sufrido una pérdida perinatal, así como dar una atención de calidad en todo este proceso.

Los estudios realizados por O'Connell et al. (2016) y Aiyelaagbe et al. (2017) recogen las opiniones de familias que han pasado por un proceso de muerte perinatal, incluyendo las pérdidas espontáneas antes y después de nacer, y las IVEs. Los resultados obtenidos muestran que los padres puntúan de forma favorable poder despedirse del bebé $(89 \%$ y $85,4 \%$ respectivamente), sostenerlo (91\% y 96,3\% respectivamente), recuerdos del bebé ( $86 \%$ y 96,4\% respectivamente) así como poder presentarlo al resto de la familia ( $86 \%$ y $86,2 \%$ respectivamente). Si bien nuestro estudio no valoró cualitativamente estos ítems, sí los valoramos a través de las preguntas sobre la calidad de atención que ofrecemos en nuestra unidad. El 100\% de los pacientes en nuestra unidad se les ofreció tener contacto con su bebé en sala de partos, aunque el 37\% manifestó que el tiempo no fue suficiente. Por otro lado, al 100\% de los pacientes se les ofreció obtener las huellas de él o ella; sin embargo, el $42 \%$ hubiera querido tener otro recuerdo como la pinza de cordón, pulsera o el gorro del bebé. En nuestra unidad actualmente no se ofrece la posibilidad realizar fotos del bebé; sin embargo, la mayoría (53\%) no le hubiera gustado recibir este recuerdo. Ante la propuesta de entregar un certificado de nacimiento a las pacientes posterior al proceso de pérdida perinatal, el $74 \%$ de las pacientes sí le hubiera gustado recibirlo.

Por otro lado, el único estudio (Cassidy, 2018) que realiza una encuesta online a 796 pacientes, mayoritariamente españolas, que habían sufrido una muerte perinatal por encima de las 16 semanas describe que el $52.9 \%$ de las madres vio al bebé, el $35.1 \%$ puedo sostener al bebé y solo el $34.7 \%$ puedo mostrar el bebé a sus familiares, mientras que el $30.4 \%$ obtuvo un recuerdo del bebé. Nuestro estudio, si bien obtuvo unos mejores resultados con respecto a la atención post mortem de la madre, solo está basado en 20 pacientes por encima de las 22 semanas en proceso de interrupción de la gestación, a diferencia del estudio de Cassidy, que es una encuesta transversal de la atención de diferentes hospitales (públicos y privados) en España. Es probable que debido a la mayor 
variabilidad de centros, la calidad de atención de las pacientes relacionadas al contacto con el bebé y los recuerdos sea menor.

Todo ello contrasta con la evidencia de diferentes estudios, como el de Crawley et al. (2013), que reportó que la oportunidad de compartir recuerdos está asociada con mejor salud mental de las pacientes. De este modo, Cacciatore, Schnebly y Froen (2009) identificaron una menor incidencia de ansiedad y depresión postparto cuando los padres recibían soporte médico posterior a la pérdida perinatal y este se asociaba a soporte emocional, disminuyendo también la probabilidad de estrés postraumático (Radestad et al., 1996; Gravensteen et al., 2013; Hughes et al., 2001). Desafortunadamente, nuestro estudio, al ser del tipo exploratorio, no valoró la relación de la entrega de recuerdos con otros factores; en cambio, sí evidenció las necesidades de nuestras pacientes debido a que $53 \%$ creía que era necesario recibir más soporte emocional o psicológico. De ellos el 20\% creía que eran mejor los grupos de apoyo, el 60\% la atención psicológica, mientras que el 20\% restantes creía útil ambas intervenciones. La mitad de los pacientes (50\%) creyó que el momento más adecuado para el apoyo emocional era al ingreso en el hospital y tras la cuarentena, mientras que el $20 \%$ solo después de terminar todo el proceso.

Por tanto, en este sentido nuestro estudio es el primero que demuestra que las pacientes españolas en proceso de pérdida perinatal necesitan soporte psicológico o de apoyo.

La calidad de la atención también implica la forma de dar las malas noticias a la paciente. Aiyelaagbe et al. (2017) describieron que la atención prestada por el obstetra fue de ayuda en un $75 \%$ de los casos, de comadronas del 92,1\%, de la comadrona especialista en pérdidas del 94,4\%, mientras que otros profesionales menos relacionados el porcentaje está por debajo del 50\%, aunque no describe quién dio la mala noticia sobre la interrupción. De forma complementaria, O’Connell et al. (2016) describen que el 47\% fue informado por los obstetras mientras que la noticia fue dada por otros profesionales el $52 \%$ de las ocasiones. El $22 \%$ de las pacientes no fueron atendidas por el grupo de duelo y 25\% no conocía que había apoyo continuo. Por su parte, Cassidy (2018) no valora estos datos.

En nuestro centro, todas las pacientes reciben el diagnóstico y la descripción del proceso de interrupción por obstetras especialistas en medicina materno-fetal. Sin 
embargo, no valoramos si este hecho fue de ayuda para las pacientes ni valoramos de forma directa la percepción que tienen ellos de otros profesionales.

La valoración en nuestro estudio se realizó en diferentes momentos del proceso de interrupción de la gestación, como son consulta obstétrica, consulta de enfermería, parto y hospitalización, teniendo como limitante el no valorar la consulta de seguimiento. Esta valoración de los diferentes ámbitos en el proceso es tan importante como la creación de recuerdos. Nuestro estudio evidencia un buena valoración (puntuación: 0-10) en general de las diferentes áreas de atención de la paciente, siendo importante el espacio $(\overline{\mathrm{x}}=8,9)$, intimidad $(\overline{\mathrm{x}}=8,36)$, confort $(\overline{\mathrm{x}}=8,19)$, ubicación de las atención $(\overline{\mathrm{x}}=7,05)$, tiempo de espera para ser atendido en consultas $(\overline{\mathrm{x}}=9,73)$ y tiempo de espera de ingreso en sala de partos $(\overline{\mathrm{x}}=8,94)$. No obstante, se obtuvo bajas puntuaciones en la ubicación de la consulta de enfermería $(\overline{\mathrm{x}}=6,05)$, así como el tiempo de espera al alta de hospitalización $(\overline{\mathrm{x}}=$ $6,22)$.

En general todos los espacios tuvieron bajas puntuaciones en la valoración de los ruidos ambientales $(\overline{\mathrm{x}}=5,98)$; este problema también fue evidenciado en las preguntas abiertas. Todo ello refleja la necesidad de ir mejorando en la atención de los pacientes al promover como ejemplo espacios aislados de ruidos y/o separados del área de atención de las gestantes o la implementación del alta precoz. Esto está acorde con Aiyelaagbe et al. (2017), que describen la importancia de tener un espacio privado (71.1\% totalmente de acuerdo).

Recientemente, con el fin de mejorar este proceso de atención, la Generalitat de Catalunya ha elaborado una guía de acompañamiento del duelo perinatal (Guasch et al., 2016) pero más enfocada al ámbito del cuidado y que podrían ser extrapoladas al cuidado de las pacientes con interrupción de la gestación.

Desafortunadamente, por el momento no existen revisiones sistemáticas que definan cuáles son estas necesidades de forma clara, y gran parte de la información disponible se ha obtenido a partir de encuestas realizadas en diferentes escenarios clínicos.

Nuestro estudio piloto es el primer estudio que valora la atención de calidad en los diferentes momentos del proceso de interrupción de la gestación y que, por tanto, da una idea de lo que se necesita cambiar y mejorar a fin de que el paciente en este proceso sienta confort e intimidad en un espacio adecuado. No obstante, nuestra encuesta tiene la 
limitación de ser una valoración numérica que, si bien trata de ser menos subjetiva, pierde la valoración relacionada a «acuerdo» o «desacuerdo» que ofrecen los pacientes.

\section{CONCLUSIÓN}

La mejora continua de la atención de los pacientes con una IVE debería ser un requisito indispensable de todos los centros de medicina materno-fetal. Para tal fin nuestro estudio implementa por primera vez una encuesta validada por distintos profesionales involucrados en el día a día de la asistencia de estas pacientes. Nuestra encuesta ha identificado diferentes puntos de mejora, lo que nos permite identificar los ítems con peores puntuaciones para tratar de buscar soluciones de forma inmediata: ubicación, ruidos ambientales, recuerdos, tiempos de espera al alta y atención psicológica.

Cabe destacar que, de forma repetida, uno de los puntos peor valorados son los ruidos ambientales en las diferentes áreas del hospital, por lo que se deduce que es necesario mejorar el aislamiento de los distintos ambientes.

En relación con el tiempo de espera al alta, parece aconsejable revisar los criterios de alta de nuestro centro para conseguir que las pacientes puedan acceder más rápidamente a un ambiente tranquilo como es el propio hogar.

Después de la realización de esta encuesta, pareció recomendable crear un grupo de soporte al duelo perinatal.

En el futuro volveremos a evaluar las mejoras obtenidas mediante un estudio del mismo formato utilizando una encuesta dirigida. 


\section{BIBLIOGRAFÍA}

AiYelaAgBe, E., et al. Assessing the quality of bereavement care after perinatal death: development and piloting of a questionnaire to assess parents' experiences. En: Journal of Obstetrics and Gynaecology. 2017, vol. 37, núm. 7, pp. 931-936.

AMERICAN COLlEge of OBSTETRICIANS AND GyneCOLIGISTS. ACOG practice bulletin No. 102: Management of stillbirth. En: Obstetrics and Gynecology. 2009, vol. 113, núm. 3, pp. 748-761.

Blood, C., \& CACCiAtore, J. Parental Grief and Memento Mori Photography: Narrative, Meaning, Culture, and Context. En: Death Studies. 2014, vol. 38, núm. 4, pp. 224233.

CACCIATORE, J. Effects of support groups on post-traumatic stress responses in women experiencing stillbirth. En: Omega: Journal of Death and Dying. 2007, vol. 55, núm.1, pp. 71-90.

Cacciatore, J.; Schnebly, S.; Froen, J. F. The effects of social support on maternal anxiety and depression after stillbirth. En: Health and Social Care in the Community. 2009, vol. 17, núm. 2, pp. 167-176.

CAssidy, P. R. Care quality following intrauterine death in Spanish hospitals: Results from an online survey. En: BMC Pregnancy and Childbirth, 2018, vol. 18, núm. 1, pp. 1-12.

CRAWley, R.; LOMAX, S.; AYERS, S. Recovering from stillbirth: The effects of making and sharing memories on maternal mental health. En: Journal of Reproductive and Infant Psychology. 2013, vol. 31, núm. 2, pp. 195-207.

DownE, S., et al. Bereaved parents' experience of stillbirth in UK hospitals: A qualitative interview study. En: BMJ Open. 2013, vol. 3, núm. 2.

ERLANDSSON, K., et al. Seeing and holding a stillborn baby: Mothers' feelings in relation to how their babies were presented to them after birth-Findings from an online questionnaire. En: Midwifery. 2013, vol. 29, núm. 3, pp. 246-250.

Flenady, V., et al. The Perinatal Society of Australia and New Zealand: Clinical Practice Guideline for Perinatal Mortality. Abril de 2009. Disponible en: https://bit.ly/3KjpiEA.

FLENADY, V., et al. Meeting the needs of parents after a stillbirth or neonatal death. En: BJOG: An International Journal of Obstetrics and Gynecology. 20147, vol. 121, pp. 137-140.

FrøEN, J. F., et al. Stillbirths: Why they matter. En: The Lancet. 2011, vol. 377, núm. 9774, pp. 1353-1366.

GóMEZ, O., et al. Interrupción legal de la gestación. Barcelona: BCNATAL y Clínic Barcelona, 2021. Disponible en: https://bit.ly/3A3Pv5x. 
Gravensteen, I. K., et al. Women's experiences in relation to stillbirth and risk factors for long-term post-traumatic stress symptoms: A retrospective study. En: BMJ Open. 2013, vol. 3, núm. 10, pp. 1-10.

GUASCH MARIA M, et al. Guía de acompañamiento al duelo perinatal. Barcelona: Generalitat de Catalunya. Departament de Salut, 2016. Disponible en: https://scientiasalut.gencat.cat/handle/11351/3323?locale-attribute=es.

HeAzELl, A. E. P., et al. Stillbirths: Economic and psychosocial consequences. En: The Lancet. 2016, vol. 387, núm. 10018, pp. 604-616.

Hughes, P., et al. Disorganised attachment behaviour among infants born subsequent to stillbirth. En: Journal of Child Psychology and Psychiatry and Allied Disciplines. 2001, vol. 42, núm. 6, pp. 791-801.

Kingdon, C., et al. Seeing and Holding Baby: Systematic Review of Clinical Management and Parental Outcomes After Stillbirth. En: Birth. 2005, vol. 42, núm. 3, pp. 206-218.

Ministerio de Sanidad, Política Social e Igualdad. Estrategia Nacional de Salud Sexual y Reproductiva. Madrid: 2011. Disponible en: https://bit.ly/3Ki1tNt.

Nuzum, D.; Meaney, S.; O'Donoghue, K. The impact of stillbirth on bereaved parents: A qualitative study. En: PLoS ONE. 2018, vol. 13, núm. 1, pp. 1-13.

O’Connell, O.; Meaney, S.; O’Donoghue, K. Caring for parents at the time of stillbirth: How can we do better? En: Women and Birth. 2016, vol 29, núm. 4, pp. 345-349.

RADESTAD, I., et al. Psychological complications after stillbirth - influence of memories and immediate management: Population based study. En: Bmj. 1996, vol. 312, núm. 7045, pp. 1505-1508. 